Neurol Med Chir (Tokyo) 49, 611 615, 2009

\title{
Isolated Recurrence of Granulocytic Sarcoma -Two Case Reports-
}

\author{
Qingsheng XU, Ming WANG, Qihan YoU*, \\ Huafeng WANG**, Ke YE, Renya ZHAN, and Yongqing ZHOU \\ Departments of Neurosurgery, ${ }^{*}$ Pathology, and ${ }^{* *}$ Hematology, the First Affiliated Hospital, \\ College of Medicine, Zhejiang University, Hangzhou, Zhejiang Province, P.R.C.
}

\begin{abstract}
Two patients presented with rare isolated recurrence of granulocytic sarcoma. A 29-year-old male presented with an extra- and intracranial mass 10 years after bone marrow transplantation for chronic myeloid leukemia. A 34-year-old female presented with an intracranial mass 3 years after complete remission of acute myeloid leukemia-M2a. Both patients underwent surgical resection. The first patient received adequate postoperative radiotherapy and chemotherapy, and achieved complete remission without evidence of diseases during the 6-month follow up. The second patient only received whole brain radiotherapy, failed to respond, and died of systemic leukemia later. These two cases demonstrate that neurosurgeons should pay attention to the occurrence of isolated recurrent granulocytic sarcoma, especially in patients with a history of hematologic neoplasm. Immediate pathological and cytogenetic diagnoses are essential.
\end{abstract}

Key words: chronic myeloid leukemia, bone marrow transplantation, acute myeloblastic leukemia, granulocytic sarcoma

Received January 23, 2009; Accepted April 24, 2009

Neurol Med Chir (Tokyo) 49, December, 2009 


\section{Introduction}

Granulocytic sarcoma (GS) is an extramedullary myeloid cell tumor (EMT), ${ }^{5,18)}$ which is known under a variety of other names in the literature, including myeloblastoma, chloroma, extramedullary myeloid tumor, and monocytic sarcoma. GS was initially described by Burns in $1811 .{ }^{4)} \mathrm{A}$ case of GS was reported as chloroma in 1853, because of its typical greenish-yellow appearance. ${ }^{11)}$ The concept of EMT, which includes isolated GS (non-leukemic GS) and leukemia with extramedullary infiltration (leukemic GS), was suggested by Davey in 1988. GS originates from a myeloid precursor and occurs as a soft tissue mass. GS may occur in a variety of tissues, including subcutaneous tissue (24\%), orbit $(24 \%)$, paranasal sinus $(11 \%)$, lymph node $(11 \%)$, bone and periosteum $(5 \%),{ }^{17)}$ and rarely the central nervous system.

The clinical status of GS has been classified into four groups: primary GS; GS identified at diagnosis of acute myeloid leukemia (AML); isolated GS without medullary relapse of AML, or a new isolated focus of GS that occurs during bone marrow remission and is not followed by medullary relapse within 30 days; and GS with concurrent bone marrow relapse of AML.5)

We describe two rare cases of isolated recurrence of extra- and intracranial GS after bone marrow transplantation in a 29-year-old male with chronic myeloid leukemia (CML), and an isolated recurrent intracranial GS in a 34year-old female after complete remission of AML-M2a.

\section{Case Reports}

Case 1: A 19-year-old male was admitted to the department of hematology in another hospital due to repeated epistaxis and gingival hemorrhage with fatigue in July 1995. Peripheral blood examination showed leukocyte count of $186,000 \mathrm{cells} / \mathrm{mm}^{3}$, hemoglobin level of $118 \mathrm{~g} / \mathrm{l}$, and platelet count of $169,000 / \mathrm{mm}^{3}$. The diagnosis was CML, based on positive testing for the B2A2 (bcr/abl) fusion gene and chromosomal analysis of the bone marrow cell confirmation of $46, \mathrm{XY}, \mathrm{Ph}(+)$. After treatment with cytarabine and hydroxyurea for one year, he was admitted to the department of hematology in our hospital for bone marrow transplantation in July 1996, which achieved complete remission.

He was readmitted to our institute in May 2005 with headache persisting for 2 months and increasing in intensity for 1 week. Physical examination found a $6 \times 6 \mathrm{~cm}$ mass above the mastoid in the left occipitotemporal region. Neurological examination was unremarkable with no focal neurological deficits and peripheral blood examination found no abnormalities. Magnetic resonance (MR) imaging showed an ellipsoidal mass about $4.11 \times 4.6 \mathrm{~cm}$ in the left occipitoparietal skull, appearing as isointense on both $\mathrm{T}_{1}$-weighted and $\mathrm{T}_{2}$-weighted imaging. The mass had invaded the left occipitoparietal lobe with adjacent brain parenchyma compression and serious edema. $\mathrm{T}_{1^{-}}$ weighted MR imaging with contrast medium showed the extra- and intracranial mass with irregular enhancement (Fig. 1).

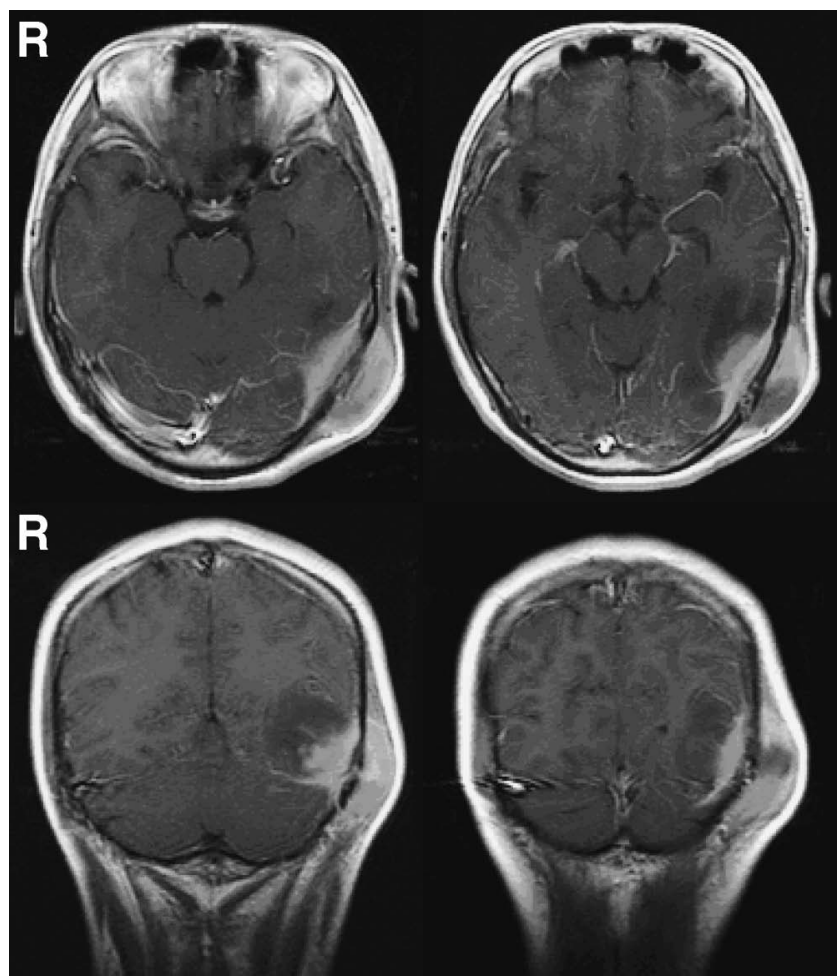

Fig. 1 Case 1. Theighted magnetic resonance images with contrast medium showing an extra- and intracranial lesion with irregular enhancement in the left occipitotemporoparietal region.

The patient underwent open surgery for the mass effect. A well-demarcated mass was found in the subcutaneous region, about $4 \mathrm{~cm}$ in diameter, which had invaded the local skull. The mass was gray and white with a friable consistency. Histological examination of the mass revealed small cell tumor, suggesting leukemic metastasis. Therefore, we only removed the whole extracranial tumor. Immunohistochemical staining demonstrated that tumor cells were positive for CD43, myeloperoxidase (MPO), CD2, CD3, and CD20, but negative for CD34. Histological examination confirmed the diagnosis of GS (Fig. 2). Postoperatively, the patient's headache was improved, and 13 days after the operation, the patient developed lymphadenectasis of the pre- and retroauricular regions. Therefore, bone marrow puncture was performed and demonstrated bone marrow manifestation of CML. Two weeks after the operation, computed tomography (CT) showed the postoperative manifestations of intracranial edema (Fig. 3). Under the guidance of the department of hematology, the patient completed radiotherapy and chemotherapy. He then recovered without deficits, and was in complete remission without evidence of disease during the 6-month follow up.

Case 2: A 34-year-old female was admitted to our institute for dizziness with walking instability and facial numbness for 1 week in December 2007, and this admission was 3 years after complete remission. The diagnosis was established as AML-M2a according to the French-American- 

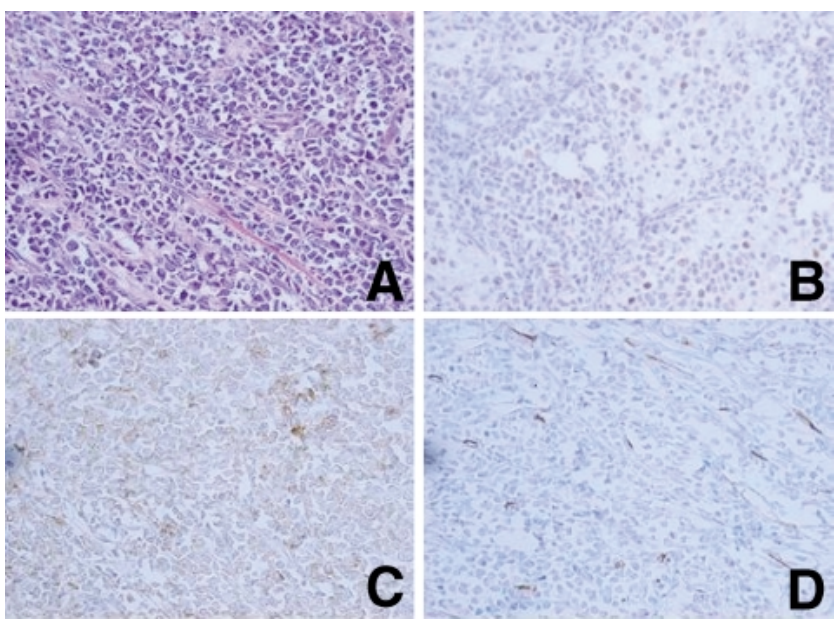

Fig. 2 Case 1. Photomicrographs of the resected tumor revealing widespread round or ovoid tumor cells infiltrating the muscles with diffused proliferation (A: hematoxylin and eosin stain, $\times 400)$, and immunohistochemical staining demonstrating that the tumor cells are positive for myeloperoxidase (B: $\times$ 400 ) and CD43 (C: $\times 400$ ), but negative for CD34 (D: $\times 400$ ).

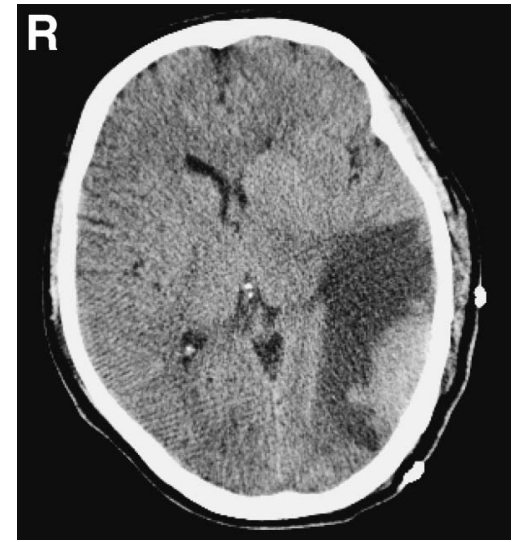

Fig. 3 Case 1. Computed tomography scan showing a high density ellipsoidal mass in the left occipital lobe, with surrounding edema, 2 weeks after the operation.

British classification in June 2003, and she had undergone two courses of systemic chemotherapy with cytarabine and harringtonine, but never achieved complete remission. She then received another two courses of chemotherapy with cytarabine, harringtonine, and aclacinomycin, which resulted in complete remission. During the interval from remission to recurrence, the patient received seven courses of systemic chemotherapy as consolidation therapy, including cytarabine, harringtonine, and aclacinomycin for two courses, middle-dose cytarabine for two courses, and cytarabine and harringtonine for another three courses. She was still in complete remission, and then she was followed up by peripheral blood examination and bone marrow examination, without any evidence of recurrence.
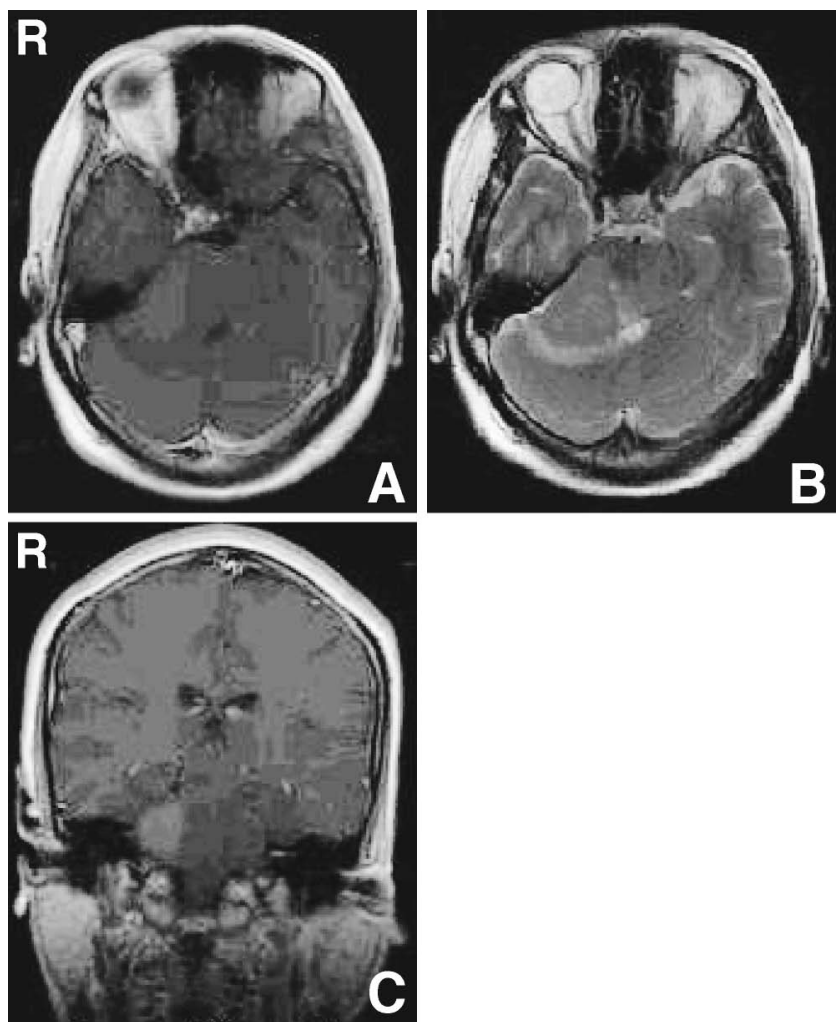

Fig. 4 Case 2. $T_{1}$-weighted magnetic resonance images showing the lesion as slightly hypointense (A), with slight homogeneous enhancement by contrast medium (C). $T_{2}$-weighted magnetic resonance image showing the lesion as isointense (B).

Physical examination found hypesthesia of trigeminal nerve on the right side of the face, right lateral hypacusis, and Romberg's sign. Pure-tone audiometry test demonstrated dysfunction of the bilateral upper brainstem auditory pathways. Peripheral blood examination found no abnormalities and bone marrow puncture examination identified M2 (bone marrow remission) without abnormal blasts. MR imaging demonstrated an irregular abnormal density mass in the right cerebellopontine angle area, appearing as slightly hypointense on $\mathrm{T}_{1}$-weighted imaging and isointense on $\mathrm{T}_{2}$-weighted imaging. $\mathrm{T}_{1}$-weighted imaging with contrast medium showed slight homogeneous enhancement and obvious occupation signs with adjacent brain parenchyma compression and slight edema, suggesting neurogenic tumor (Fig. 4).

The patient underwent open surgery. Immunohistochemical staining demonstrated that the tumor cells were mainly positive for CD43, CD34, CD117, and MPO. The histological diagnosis was GS (Fig. 5). The patient received whole brain radiotherapy, but showed no response, and died of systemic leukemia 11 months after the operation.

\section{Discussion}

Reports of isolated recurrent intracranial or extra- and in- 


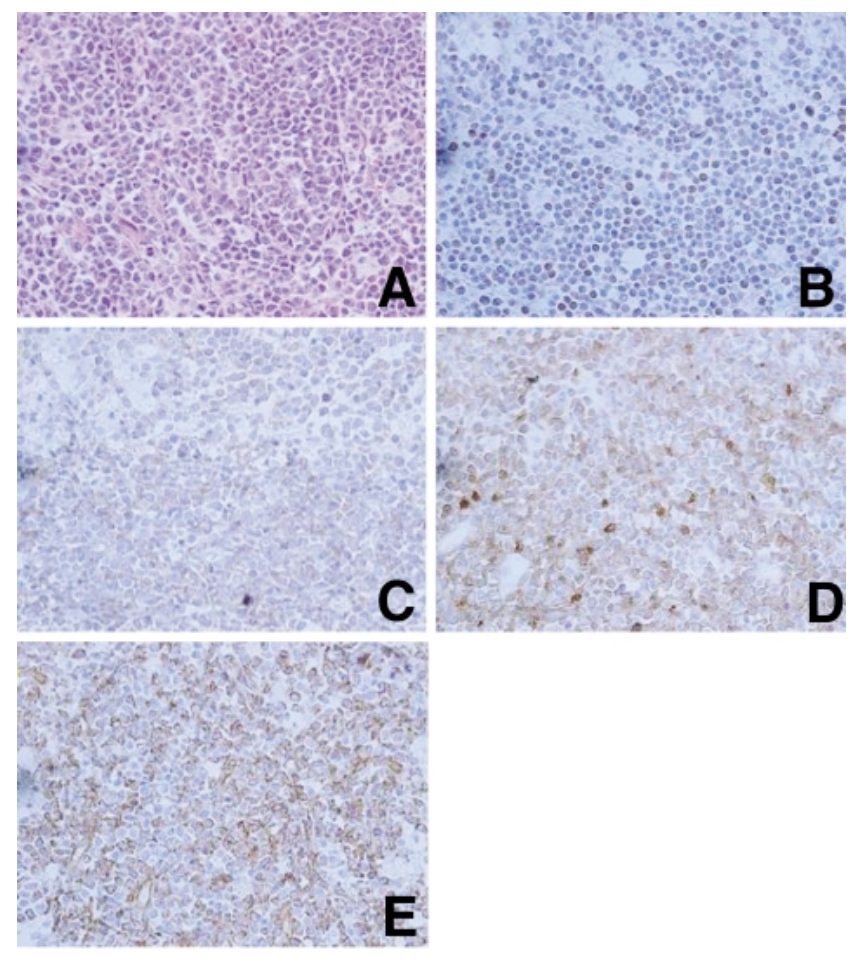

Fig. 5 Case 2. Photomicrographs of the resected tumor revealing the tumor consisting of dominant large cells, which are widespread and diffusely proliferate (A: hematoxylin and eosin stain, $\times 400$ ), and immunohistochemical staining demonstrating that the tumor cells are positive for myeloperoxidase (B: $\times$ 400), CD43 (C: $\times 400)$, CD117 (D: $\times 400)$, and CD34 (E: $\times 400)$.

tracranial GS are relatively rare, and most are sporadic case reports. The clinical status of GS is usually associated with hematologic neoplasms, such as AML, myeloproliferative disorder, and myelodysplastic syndrome. The majority of GS cases were associated with AML, especially AML-M2. A 30-year-old female presented with isolated recurrence of GS manifesting as extra- and intracranial masses 16 months after complete remission of M2.13) A 34year-old female with AML-M3 in remission developed unilateral hearing loss caused by cerebellopontine angle GS identified by MR imaging 27 months after remission, but histological examination of the GS specimen revealed AML-M4. ${ }^{8}$ A 45-year-old male patient diagnosed with AML-M4 who remained in complete remission for 16 years, then a large subdural GS was found, and the final histological examination showed AML-M0.17) A 41-yearold man with AML-M7 developed GS in the right occipital lobe after complete remission. ${ }^{14)}$ Other types of AML, i.e. M1, M5, and M6, have not been found with isolated recurrence of intracranial or extra- and intracranial GS. Isolated recurrence of intracranial GS was also reported in patients with other hematologic neoplasms. A case of intracranial GS associated with eosinophilia developed in the process of myelofibrosis was reported.6) The present highly unusual case of isolated recurrence of extra- and intracranial GS after bone marrow transplantation occurred in a 29-year-old female with CML.
The clinical manifestations of isolated recurrence of intracranial or extra- and intracranial GS are similar. Both occur as intracranial neurogenic tumors, with symptoms of intracranial hypertension, skull destruction, and absence of systemic symptoms. Laboratory findings are normal. CT and MR imaging findings only provide indirect evidence for the diagnosis. Isolated recurrence of intracranial GS usually appears as an intracranial round or roughly round high-density mass on CT or MR imaging. ${ }^{19)}$ Sometimes, GS appears similar to malignant lymphoma in iconography. The GS looked like neurogenic tumor on MR imaging in our second case. Therefore, the cytological and histological diagnoses are determinative.

Positive MPO staining is an especially important indication. Positive staining for MPO, CD20 (L26), CD4 (Leu22), and CD68 can identify 96\% of cases of EMT. ${ }^{18 \text { ) }}$ CD43 is the only antigen expressed in all EMT. CD34 is expressed in $30 \%$ of GS and $60 \%$ of blast type GS, and can help to establish the differential diagnosis between GS and lymphoma. ${ }^{2)}$ However, positive CD34 is not necessary for all GS. ${ }^{3)}$ A case of GS of the epididymis was negative for CD34. ${ }^{16)}$ CD34 was positive in only one of our two cases. Approximately $75 \%$ of EMT express CD45 (leukocyte common antigen). CD3 expression rate is $36 \%$ and CD20 is rarely expressed. The expression level of neural cell-adhesion molecules (NCAM) may be higher in AML patients with $t(8 ; 21) .{ }^{9)}$ NCAM is found in the brain, neural, muscle, and natural killer cells, which tends to target neoplasia in particular types. ${ }^{7)}$ Therefore, $t(8 ; 21)$ and expression of NCAM may be related to extramedullary infiltration of GS.

The prognosis for GS is not optimistic. Primary GS may transform to acute leukemia in 8 days to 28 months. ${ }^{15)}$ However, review of 72 cases of non-leukemic GS suggested that the time of transformation was 10 to 12 months. ${ }^{21)}$ Even the development of GS in patients with leukemia almost always heralds an impending blast crisis. Experience with therapy for GS is limited, with no experimental findings available.

Chemotherapy combined with radiation is still the treatment of first choice for isolated intracranial GS. ${ }^{20}$ Anti-leukemic chemotherapy containing high-dose cytarabine might provide good results. ${ }^{10)}$ Cytology and histology can identify the pathological classification and provide guidance for chemotherapy. The effects of surgery remain controversial. Surgical decompression may provide rapid results, and improve the quality of survival. Surgery is indicated in the presence of progressive neurological deficit. ${ }^{12)}$ Removal of an early-stage tumor may lead to a long symptom-free period, and good responses to external radiation have also been documented. ${ }^{1,12)}$ However, surgical resection and/or irradiation may only achieve local control, with no influence on survival. On the other hand, surgery could increase the risk of infection and central nervous system dissemination, so there is no advantage over chemotherapy and irradiation. ${ }^{13)}$

The present two cases demonstrate that neurosurgeons should pay attention to the occurrence of isolated recurrence of intracranial or extra- and intracranial GS, especially in patients with a history of hematologic neoplasms. 
Immediate pathological and cytogenetic diagnoses are essential for better outcomes. Further research is needed to improve and standardize the therapy for GS.

\section{References}

1) Ahn JY, Kwon SO, Shin MS, Kang SH, Kim YR: Meningeal chloroma (granulocytic sarcoma) in acute lymphoblastic leukemia mimicking a falx meningioma. J Neurooncol 60: 31-35, 2002

2) Astall E, Yarranton H, Arno J, Marcus R: Granulocytic sarcoma preceding AML M0 and the diagnostic value of CD34. J Clin Pathol 52: 705-707, 1999

3) Audouin J, Comperat E, Le Tourneau A, Camilleri-Broet S, Adida C, Molina T, Diebold J: Myeloid sarcoma: clinical and morphologic criteria useful for diagnosis. Int J Surg Pathol 11: 271-282, 2003

4) Burns A: Observations on the Surgical Anatomy of the Head and Neck, ed 1. Edinburgh, Thomas Bryce \& Co, 1811, pp 364-366

5) Byrd JC, Edenfield WJ, Shields DJ, Dawson NA: Extramedullary myeloid cell tumors in acute nonlymphocytic leukemia: a clinical review. J Clin Oncol 13: 1800-1816, 1995

6) Chan SW, Datta NN, Thomas TM, Chan KW: Intracranial chloroma in hypereosinophilic myelofibrosis. Surg Neurol 59: $55-57,2003$

7) Cunningham BA, Hemperly JJ, Murray BA, Prediger EA, Brackenbury R, Edelman GM: Neural cell adhesion molecule: structure, immunoglobulin-like domains, cell surface modulation, and alternative RNA splicing. Science 236: 799-806, 1987

8) Gokcan MK, Batikhan $\mathrm{H}$, Calguner M, Tataragasi AI: Unilateral hearing loss as a presenting manifestation of granulocytic sarcoma (chloroma). Otol Neurotol 27: 106-109, 2006

9) Hurwitz CA, Raimondi SC, Head D, Krance R, Mirro J Jr, Kalwinsky DK, Ayers GD, Behm FG: Distinctive immunophenotypic feature of $\mathrm{t}(8 ; 21)(\mathrm{q} 22 ; \mathrm{q} 22)$ acute myeloblastic leukemia in children. Blood 80: 3182-3188, 1992

10) Huter O, Brezinka C, Nachbaur D, Schwaighofer H, Lang A, Niederwieser D: Successful treatment of primary extramedullary leukemia (EML) of the uterus with radical therapy, chemotherapy, autologous bone marrow transplantation (BMT) and prophylactic local irradiation. Bone Marrow
Transplant 18: 663-664, 1996

11) King A: A case of chloroma. Monthly J Med 17: 97, 1853

12) Lee SH, Park J, Hwang SK: Isolated recurrence of intracerebral granulocytic sarcoma in acute lymphoblastic leukemia: a case report. J Neurooncol 80: 101-104, 2006

13) Nishimura S, Kyuma Y, Kamijo A, Maruta A: Isolated recurrence of granulocytic sarcoma manifesting as extra- and intracranial masses - case report. Neurol Med Chir (Tokyo) 44: 311-316, 2004

14) Obara $H$, Nishimura $S$, Hayashi $N$, Numagami $Y$, Inoue $T$, Kubo K, Kaimori M, Nishijima M: [Intracranial granulocytic sarcoma in a patient with acute myeloid leukemia]. No To Shinkei 58: 797-801, 2006 (Jpn, with Eng abstract)

15) Pathak B, Bruchim I, Brisson ML, Hammouda W, Bloom C, Gotlieb WH: Granulocytic sarcoma presenting as tumors of the cervix. Gynecol Oncol 98: 493-497, 2005

16) Sarvis JA, Auge BK: Myeloid (granulocytic) sarcoma of epididymis as rare manifestation of recurrent acute myelogenous leukemia. Urology 73: 1163.e1-1163.e3, 2009

17) Smidt $M H$, de Bruin HG, van't Veer MB, van den Bent MJ: Intracranial granulocytic sarcoma (chloroma) may mimic a subdural hematoma. J Neurol 252: 498-499, 2005

18) Traweek ST, Arber DA, Rappaport H, Brynes RK: Extramedullary myeloid cell tumors. An immunohistochemical and morphologic study of 28 cases. Am J Surg Pathol 17: 1011-1019, 1993

19) Wright $\mathrm{DH}$, Hise JH, Bauserman SC, Naul LG: Intracranial granulocytic sarcoma: CT, MR, and angiography. J Comput Assist Tomogr 16: 487-489, 1992

20) Yamamoto $K$, Hamaguchi $H$, Nagata $K$, Hara $M$, Tone $O$, Tomita $\mathrm{H}$, Ito U: Isolated recurrence of granulocytic sarcoma of the brain: successful treatment with surgical resection, intrathecal injection, irradiation and prophylactic systemic chemotherapy. Jpn J Clin Oncol 29: 214-218, 1999

21) Yamauchi K, Yasuda M: Comparison in treatments of nonleukemic granulocytic sarcoma: report of two cases and a review of 72 cases in the literature. Cancer 94: 1739-1746, 2002

Address reprint requests to: Yongqing Zhou, M.D., Department of Neurosurgery, the First Affiliated Hospital, College of Medicine, Zhejiang University, No. 79 Qingchun Road, Hangzhou, Zhejiang 310003, P.R.C. 